\title{
IMPACT OF A PHARMACIST-LED EDUCATIONAL INTERVENTION ON QUALITY OF LIFE AMONG PATIENTS WITH ASTHMA
}

\author{
QUYNH THI HUONG BUI ${ }^{1 *}$, KHOI XUAN PHAM ${ }^{1,2}$, TIEN HOANG TRAN ${ }^{3}$, LAN THI TUYET LE ${ }^{4}$, HO NHU NGUYEN ${ }^{1}$
}

${ }^{1}$ Department of Clinical Pharmacy, Faculty of Pharmacy, University of Medicine and Pharmacy, Ho Chi Minh City, Vietnam. ${ }^{2}$ Department of Clinical Pharmacy, Faculty of pharmacy, Lac Hong University, Dong Nai Province, Vietnam. ${ }^{3}$ Department of Pharmacy, University Medical Center, Ho Chi Minh City, Vietnam. ${ }^{4}$ Department of Screening Respiratory Function, University Medical Center, Ho Chi Minh City, Vietnam. Email: bthquynh@ump.edu.vn

Received: 23 April 2019, Revised and Accepted: 13 May 2019

\section{ABSTRACT}

Objectives: Asthma is a chronic disease resulting in reduced quality of life (QoL) of most affected individuals. Training in asthma-related knowledge, inhaler skills, medicine usage, and the prevention of adverse drug events is demonstrated to improve asthma patients' QoL. Therefore, the research was conducted to evaluate the effectiveness of education intervention undertaken by pharmacists on QoL of patients with asthma.

Methods: We conducted a clinical randomized controlled trial among asthmatic outpatients aged 18 years or older in the Department of Screening Respiratory Function, University Medical Center, Ho Chi Minh City, Vietnam. Patients were randomized into an intervention group (IG) and a non-IG (NIG). The clinical pharmacists' intervention program included training in asthma-related knowledge, inhaler technique, recognition, prevention of adverse drug events, and lifestyle adjustment. Participant screening was conducted using the telephone on a monthly basis. QoL was measured using the Vietnamese Version of the Mini Asthma QoL Questionnaire (MiniAQLQ). The main outcome was the difference in QoL scores between IG and NIG after 3 months follow-up.

Results: After 3 months, the number of patients with asthma in the IG and in the NIG was 173 and 96, respectively. QoL mean scores in the IG patients were significantly higher than those in their NIG counterparts ( $1.79 \pm 1.01$ vs. $1.06 \pm 0.93$, respectively, $\mathrm{p}<0.001)$. Pharmacist-based interventions overall QoL scores (multivariate-adjusted regression coefficient $=0.362 ; \mathrm{p}<0.001$ ).

Conclusions: Clinical pharmacist-led counseling can improve asthmatic patients' QoL.

Keywords: Quality of life, Clinical pharmacist-led intervention, Mini Asthma Quality of life Questionnaire, Randomized controlled trial, Asthmatic education.

(c) 2019 The Authors. Published by Innovare Academic Sciences Pvt Ltd. This is an open access article under the CC BY license (http://creativecommons. org/licenses/by/4. 0/) DOI: http://dx.doi.org/10.22159/ajpcr.2019.v12i6.33705

\section{INTRODUCTION}

The worldwide prevalence of asthma has increased rapidly [1]. Growing attention has been drawn to Asia, including Vietnam where the prevalence of asthmatic adults was reported to range from $2.5 \%$ to $5.0 \%$ [2]. Patients with asthma can suffer from critical health problems such as reduced quality of life (QoL) and increased risk of death [3-5]. Of those approaches to improve QoL among asthma patients, pharmacistled educational interventions in collaboration with physicians (e.g., face-to-face or email counseling providing information of asthma, medication management, and lifestyle changes) have been demonstrated to be applicable to improve safety, effectiveness of treatment outcomes, and QoL of patients with long-term medical condition [6,7], such as asthma [8-10]. However, those approaches are not widely implemented across the health-care system in Vietnam. Therefore, this study aimed to evaluate whether asthma patients gain health benefits from pharmacistled educational interventions involving face-to-face and telephone counseling in a hospital in Vietnam.

\section{METHODS}

\section{Setting and study participants}

We conducted a randomized controlled clinical trial of outpatients who were diagnosed with asthma (International Classification of Diseases 10:J45), aged $\geq 18$ years visiting Department of Respiratory Function Screening (DRFS), University Medical Center, from October 15, 2016, to February 15, 2017. Participants were excluded if (1) personal information or contact addresses were incomplete; (2) Mini-Mental State Examination (MMSE) scores were <17; (3) baseline mini asthma qol questionnaire (MiniAQLQ) scores were $\geq 6$; and (4) patients were diagnosed with chronic obstructive pulmonary disease (COPD) or (5) illiterate.

\section{Baseline examination}

Baseline characteristics were collected for all participants. Demographic data included age, gender, and education level. For baseline examination, participants were asked to wear light clothing and no shoes before measuring body height and weight. Body mass index (BMI) is defined as the weight $(\mathrm{kg})$ divided by the square of height $(\mathrm{m})$. Obese patients were those with $\mathrm{BMI} \geq 25 \mathrm{~kg} / \mathrm{m}^{2}$ and overweight patients were those with BMI range from $23 \mathrm{~kg} / \mathrm{m}^{2}$ to $25 \mathrm{~kg} / \mathrm{m}^{2}$ according to international obesity taskforce classification [11]. Data on asthma at baseline included asthma severity (based on GINA 2016 classification) [12], having any comorbidities, having asthma-related comorbidities (including gastroesophageal reflux disease [GERD], allergic rhinitis, and allergic condition), and mistake in inhaler technique (defined as "Yes" if a participant had at least one incorrect step of inhaler usage and "No" if all steps were correct; the technique instructions followed GINA 2016) [12].

\section{QoL assessment}

We used a validated Vietnamese version of the MiniAQLQ to calculate QoL scores for all patients [13]. The MiniAQLQ questionnaire includes 15 questions classified into four main domains: Symptom (questions $1,4,6,8$, and 10), activity (questions 12,13,14, and 15), environment (questions 2, 7, and 11), and emotion (questions 3, 5, and 9). Each question is given a score ranging from 1 to 7 . A higher score denotes 
a higher QoL; an increase in the total score indicates an improvement in QoL [14]. Every patient would complete two surveys about QoL, one at the beginning of the study and one at the end of follow-up for about 10 min each time.

\section{Intervention program}

Eligible patients were randomly allocated into the IG or the control group (CG) using a list composed by Excel corresponding with their registered order at DRFS. Both groups were interviewed about their inhaler techniques and QoL at baseline during a face-to-face meeting and at the end of the study by mobile phone call. Patients in the CG only received usual care from health-care providers and were not aware of the ongoing intervention, whereas those in the IG were additionally consulted by clinical pharmacists repeatedly. At the end of the patients' first visit to the outpatient department, the clinical pharmacist educated the IG on medication self-management. Patients were shown diseaserelated knowledge, how to use inhalers correctly, how to prevent or handle the common adverse drug effects, and some advice on lifestyle changes. The content of the educational session was based on guidelines from the Global Initiative for Asthma 2016 (GINA 2016) [12]. Next, every month, clinical pharmacists made a mobile phone call to ensure IG patients had followed the instructions correctly. At the end of the follow-up, the researchers reassessed QoL scores in both groups. For ethnicity purposes, CG patients were also given a short counseling period on disease and proper drug use after the survey finished. The research process is shown in Fig. 1.

\section{Outcomes}

The main outcome was the differences in QoL score changes from baseline after the 3-month intervention between the IG and the CG.

\section{Sample size}

The required sample size for each group was calculated using the following formula:

$$
\mathrm{n}=\frac{2 \mathrm{C}}{\mathrm{ES}^{2}}=\frac{2 \mathrm{C}}{\left(\frac{\mu_{1}-\mu_{2}}{\mathrm{~s}_{1}}\right) 2}
$$

where $C=7.85(\alpha=0.05$, reliability 95\%, $\beta=0.2$, and power=0.8). According to Bereznicki et al. [15], each group should have a minimum size of 90 participants. To account for the loss of follow-up, we recruited at least 110 patients for each group.

\section{Statistical analysis}

Descriptive data were presented as mean and Standard deviation if normally distributed or median and IQR if skewed. Differences between two groups were evaluated using Chi-square tests for percentages or t-test for mean values. The association between intervention program and QoL was assessed using multivariate regression analysis, adjusted for potential confounders including baseline age $(18-60 ; \geq 60$ years of age), gender (male; female), overweight/obesity (yes; no), asthma severity grade (from 1 to 5), education level (primary/secondary/ high school level; college/university/postgrad-level), having any comorbidities (yes; no), having GERD/allergic rhinitis/Allergic (yes; no), improper inhaler technique (yes; no), and baseline MiniAQLQ scores. Results were presented as per-protocol analysis for the remaining sample after 3-month follow-up. We also considered an intention-totreat (ITT) analysis with baseline characteristics of all participants at randomization. In case a patient was lost to follow-up, we assumed that the QoL at the 3-month interval was unchanged. Statistical analyses were performed using the Statistical Package for the Social Sciences Program, version 20.0. The level of statistical significance was specified at $\mathrm{p}<0.05$.

\section{Ethics approval}

Informed consent was obtained from all study participants. The protocol was approved by the Institutional Review Board of University

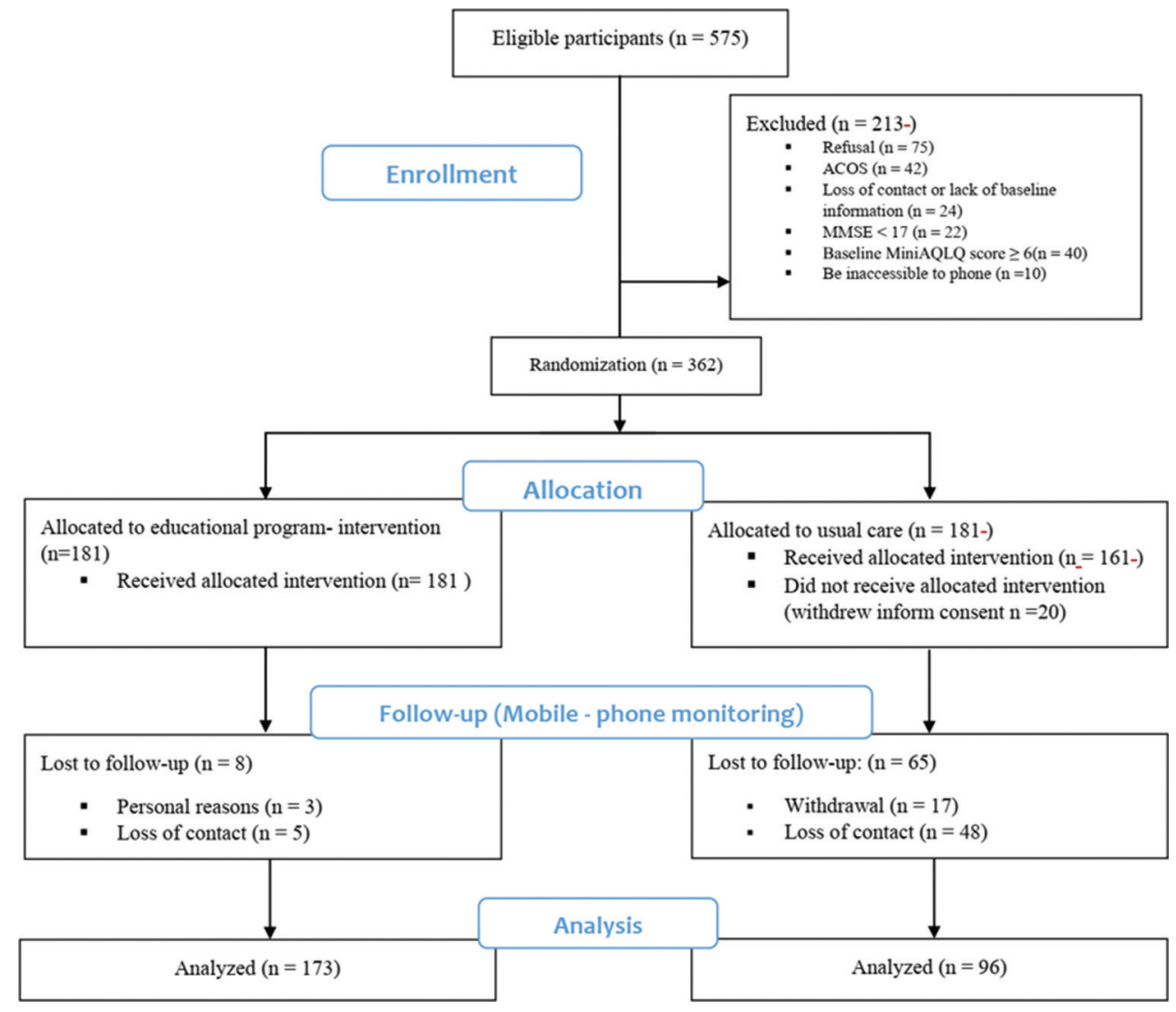

Fig. 1: Flowchart of the research process 
Medical Center, Ho Chi Minh City, Vietnam. All procedures in this study followed the ethical standards of the institutional and/or national research committee and all amendments or comparable ethical standards, including the 1964 Helsinki declaration.

\section{RESULTS}

A number of 575 patients were eligible to enter the study. Participants were excluded if they refused to take part in the study ( $n=75)$, had asthma - COPD overlap syndrome (ACOS) $(n=42)$, had missing baseline information $(n=24)$, were inaccessible to phone $(n=10)$, had MMSE scores $<17(n=22)$, or baseline MiniAQLQ score $\geq 6(n=40)$. The resulting sample left a total of 362 patients for randomization (181 in IG and 181 in CG). After the 3-month follow-up, 269 patients remained in the study (Fig. 1). Results are presented as per protocol analysis on the remaining participants.

\section{Patient characteristics}

All characteristics at baseline for all patients in IG and CG are summarized in Table 1 . Most patients were in the middle age $(85.5 \%$ [IG] vs. $87.5 \%$ [CG]). The majority was female (61.8\% [IG] vs. 66.7\% [CG]) and having education level lower than college (63\% [IG] vs. 67.8\% [CG]). The allergic rhinitis was the most prevalent comorbidity $(75.8 \%$ [IG] vs. $69.7 \%$ [CG]). A substantially high number of patients had at least one error in inhaler techniques (91.9\% [IG] vs. 91.5\% [CG]).

\section{Assessment of QoL}

Baseline MiniAQLQ scores are presented in Table 2. Except for the environment, the scores for the other three domains and overall score between the two groups were not statistically different $(p>0.05)$.
After 3 months, the overall QoL scores in the IG were significantly higher than those in the CG $(6.10 \pm 0.84$ vs. $5.50 \pm 0.98, \mathrm{p}<0.001)$. A statistically significant difference in changes of scores from baseline was also observed in patients receiving pharmacist-led intervention compared with those in the CG (overall changes of score $1.79 \pm 1.01$ vs. $1.06 \pm 0.93$, $\mathrm{p}<0.05)$.

In a multivariate linear regression model, the significance was still held after adjusting for confounding factors including age, sex, education level, obesity, comorbidities, and baseline inappropriate inhaler technique $(\mathrm{p}<0.001)$ (Table 3$)$.

To investigate whether or not the large loss to - follow-up proportion (>20\%) affected our findings, we performed two analyses. In the first one, referred as ITT analysis, results of multivariate regression analysis in all patients at randomization $(n=342)$ showed that intervention program was associated with a positive change in QoL ( $\beta=0.474 ; 95 \%$ Confidence interval: $0.830 \div 1.214 ; \mathrm{p}<0.001$ ) (Supplemental Table 1 ). In the second analysis, we assessed if there were any differences in the baseline characteristics between remaining CG patients and loss to follow-up CG patients after 3 months. No, statistically differences in baseline demographic features or MiniAQLQ scores were detected (Supplemental Table 2).

\section{DISCUSSION}

The current study showed that a pharmacist-led educational intervention improved QoL in patients with asthma after a short period (3 months). The MiniAQLQ scores and its changes $(\Delta)$ in the IG were significantly higher than those in CG $(\mathrm{p}<0.001)$. Furthermore, the results of the multivariate regression analysis confirmed the major

Table 1: Baseline characteristics of participants $(n=269)$

\begin{tabular}{|c|c|c|c|c|}
\hline \multirow[t]{2}{*}{ Characteristics } & \multirow[t]{2}{*}{ Overall (\%) } & \multicolumn{2}{|l|}{ Group } & \multirow[t]{2}{*}{ p-value } \\
\hline & & IG $(n=173)(\%)$ & CG $(n=96)(\%)$ & \\
\hline Female & 65.8 & 61.8 & 66.7 & 0.342 \\
\hline Mean age & $42.02 \pm 14.95$ & $40.92 \pm 15.49$ & $43.27 \pm 13.36$ & 0.161 \\
\hline $18-60$ & 85.1 & 85.5 & 87.5 & 0.656 \\
\hline$\geq 60$ & 14.9 & 14.5 & 12.5 & \\
\hline BMI $\left(\mathrm{kg} / \mathrm{m}^{2}\right)$ & $22.32 \pm 2.69$ & $22.09 \pm 2.58$ & $22.95 \pm 3.04$ & 0.034 \\
\hline Over-weight/obesity & 37.7 & 37 & 46.9 & 0.114 \\
\hline \multicolumn{5}{|l|}{ Education level } \\
\hline Primary/secondary/high school & 66.1 & 63 & 67.8 & 0.257 \\
\hline University/college/postgraduate & 33.9 & 37 & 32.2 & \\
\hline Asthma severity (GINA 2016) & $3.18 \pm 0.93$ & $3.16 \pm 0.85$ & $3.10 \pm 0.99$ & 0.938 \\
\hline \multicolumn{5}{|l|}{ Comorbidities } \\
\hline Yes & 86 & 87 & 85.4 & 0.730 \\
\hline GERD & 29.9 & 36.6 & 29.2 & 0.235 \\
\hline Allergic rhinitis/sinusitis & 71.5 & 75.8 & 69.7 & 0.294 \\
\hline
\end{tabular}

Mean age, body mass index, and asthma were compared between the two groups using the t-test. Rates of gender, age groups, overweight/obesity patients, education level, comorbidities, and inappropriate inhaler technique were compared using the Chi-square test. BMI: Body mass index, GERD: Gastroesophageal reflux disease

Table 2: QoL score at baseline and after 3 months follow-up (n=269)

\begin{tabular}{|c|c|c|c|c|c|c|c|c|c|}
\hline \multirow[t]{2}{*}{ Characteristics } & \multicolumn{3}{|c|}{ Baseline QoL scores } & \multicolumn{3}{|c|}{ After 3 months QoL scores } & \multicolumn{3}{|c|}{ Changes of QoL scores ( $\Delta$ score $^{*}$ ) } \\
\hline & IG & CG & p-value & IG & CG & p-value & IG & CG & p-value \\
\hline MiniAQLQ score & $4.31 \pm 0.89$ & $4.45 \pm 0.92$ & 0.170 & $6.10 \pm 0.84$ & $5.50 \pm 0.98$ & $<0.001$ & $1.79 \pm 1.01$ & $1.06 \pm 0.93$ & $<0.001$ \\
\hline Symptom score & $4.00 \pm 1.16$ & $4.21 \pm 1.23$ & 0.109 & $6.31 \pm 0.74$ & $5.51 \pm 1.20$ & $<0.001$ & $2.31 \pm 1.29$ & $1.30 \pm 1.27$ & $<0.001$ \\
\hline Activity score & $5.29 \pm 1.12$ & $5.07 \pm 1.30$ & 0.265 & $6.36 \pm 0.74$ & $5.64 \pm 1.10$ & $<0.001$ & $1.07 \pm 1.15$ & $0.58 \pm 1.06$ & 0.001 \\
\hline Environment score & $3.99 \pm 1.44$ & $4.42 \pm 1.45$ & 0.010 & $5.54 \pm 1.54$ & $5.39 \pm 1.56$ & 0.241 & $1.55 \pm 1.27$ & $0.97 \pm 1.39$ & 0.002 \\
\hline Emotion score & $3.86 \pm 1.37$ & $4.05 \pm 1.21$ & 0.232 & $5.98 \pm 1.19$ & $5.43 \pm 1.34$ & $<0.001$ & $2.12 \pm 1.56$ & $1.38 \pm 1.34$ & 0.001 \\
\hline
\end{tabular}

Mean MiniAQLQ score, Symptom score, Activity score, Environment score, Emotion score, and the changes of MiniAQLQ score and all other domains of IG and CG were compared using the t-test. QoL: Quality of Life, MiniAQLQ: Mini Asthma QoL Questionnaire 
Table 3: Effect of an intervention program on total QoL scores after 3-month follow-up

\begin{tabular}{llllc}
\hline Factor & Beta & $\mathbf{9 5 \%}$ CI & \multicolumn{1}{c}{ p-value } \\
\cline { 3 - 4 } & & Upper & Lower \\
\hline Intervention (yes) & & 0.514 & 0.924 & $<0.001$ \\
Baseline QoLscore & 0.362 & 0.330 & 0.545 & $<0.001$ \\
Age & 0.015 & -0.008 & 0.006 & 0.776 \\
Gender (Male) & -0.047 & -0.297 & 0.112 & 0.375 \\
Education level (University/College/Postgrad) & 0.046 & -0.136 & 0.316 & 0.433 \\
Allergic rhinitis/sinusitis (yes) & 0.060 & -0.192 & 0.447 & 0.432 \\
GERD (yes) & -0.072 & -0.382 & 0.092 & 0.228 \\
Allergy (yes) & 0.222 & 0.224 & 0.673 & $<0.001$ \\
Having any comorbidity (yes) & -0.006 & -0.445 & 0.411 & 0.938 \\
Asthma severity & 0.213 & 0.113 & 0.337 & 0.001 \\
Over weight/Obesity (yes) & 0.035 & -0.136 & 0.271 & 0.513 \\
Baseline inappropriate inhaler technique & 0.065 & -0.129 & 0.569 & 0.218 \\
\hline
\end{tabular}

GERD: Gastroesophageal reflux disease, CI: Confidence interval

contribution of the intervention factor in increasing QoL scores despite the apparent role of the patient's allergy situation and asthma severity.

Our findings are consistent with four previous studies in which interventions were direct educational programs, face-to-face meetings with patients, or only providing instructions through email and information package [15-18]. In these studies, the QoL scores in the IG were significantly higher than those in the CG. In contrast, two other studies reported different findings from our study $[19,20]$. Although these studies were unable to demonstrate the effect of education-based interventions, several positive findings were recorded. For instance, Wang et al. indicated that symptom scores in the IG significantly increased after 6-month follow-up [19]; Petkova showed that QoL scores in the IG increased while those in the CG decreased after 4 months [20].

Besides the disparity in some demographic characteristics, our distinction from the previous studies was the higher frequency of patient-pharmacist contact and intervention approach. Particularly, the interval between consultations was every 2 and 3 months or longer period of 6 months or 12 months in studies by Plaza et al., Armour et al., and Wang et al. $[16,17,19]$, whereas in the studies by Bereznicki et al. [15] and Shanmugam et al. [18], there was only one consultation at the beginning of the study. However, in the current study, clinical pharmacists communicated with patients at the start of the study and repeatedly at every month. In general, communication between healthcare providers and patients in consultations is the keystone to improve the effectiveness of therapy $[10,21,22]$. Good pharmacist-patient communication or consultation can help to increase asthma control, belief in pharmacotherapy, inhaler-usage [22-24]. Therefore, repeated consultation for patients with asthma may optimize their inhaler usage skills and consequently, increase the patient's QoL [23].

Another strength of our study is a combination of face-to-face and mobile phone counseling. Despite lacking previous supporting research for the benefit of this combination in improving asthmatic patients' QoL, each type of these approaches has been positively demonstrated to facilitate patients' treatment [8-10,25-29] Particularly, face-to-face consultations may raise patients' awareness of asthma and enhance their proper use of inhalers [8-10,25]. In some studies, counseling and patient monitoring through phone were shown to be feasible for not only following up a large number of patients but also supporting patients in asthma control and medicine usage [26-29]. Moreover, most of our patients visited the hospital from distant provinces; thus, the mobile phone was an available option to contact patients for consultation when they were back home. That is why it was reasonable for us to combine two methods in the educational program of which purpose is to improve the patient's QoL.

Last but not least, our study provided evidence to support the key role of pharmacists in improving issues among patients with asthma. Together with physicians, pharmacists could monitor the treatment process; provide useful advice on medicine usage and lifestyle changes to asthmatic patients [30-31]. This perception and our result may give more evidence for strengthening Vietnam's clinical pharmacy system, which is still lacking facilities and standard clinical pharmacists [32].

\section{Limitation}

Our study had some limitations. First, the percentage of loss to follow-up after 3-months was above $20 \%$ in the CG. However, the baseline characteristics between patients lost to follow-up and the remaining participants after 3 months were not statistically different, and the ITT analysis showed similar results with per protocol analysis (Supplemental Table 1 and 2). Second, the duration of follow-up was not long enough to verify the permanent effects of the intervention. Finally, the study was also designed as an open trial, which could reduce reliability due to the interference of objective elements (such as viewpoints of interviewers or dishonesty of patients). Therefore, a longer blinded trial may be necessary to overcome this issue.

\section{CONCLUSION}

The pharmacist-led educational intervention integrating face-to-face consultation and mobile phone counseling was demonstrated to increase QoL of asthmatic patients. It may be necessary to standardize these programs as routine health care to improve the quality of treatment.

\section{ACKNOWLEDGMENTS}

The authors thank all the staff members of the Screening Respiratory Function Department, University Medical Center, Ho Chi Minh City, for granting permission and helping the research group throughout the study process.

\section{AUTHORS'CONTRIBUTIONS}

Quynh Thi Huong Bui, Khoi Xuan Pham, Tien Hoang Tran, Ho Nhu Nguyen, and Lan Thi Tuyet Le conceived and designed the study. Khoi Xuan Pham, Tien Hoang Tran, and Quynh Thi Huong Bui performed the study. Quynh Thi Huong Buiand Khoi Xuan Pham analyzed the data. Quynh Thi Huong Bui, Khoi Xuan Pham wrote the paper. Quynh Thi Huong Bui, Ho Nhu Nguyen, and Lan Thi Tuyet Le revised the manuscript for important intellectual content. All authors read and approved the final manuscript.

\section{CONFLICTS OF INTEREST}

All authors approved the manuscript and this submission. The authors reported no conflicts of interest and no funding was received for this work.

\section{REFERENCES}

1. GBD Chronic Respiratory Disease Collaborators. Global, regional, and national deaths, prevalence, disability-adjusted life years, and years lived with disability for chronic obstructive pulmonary disease and asthma, 1990-2015: A systematic analysis for the global burden of 
disease study 2015. Lancet Respir Med 2017;5:691-706.

2. Song WJ, Kang MG, Chang YS, Cho SH. Epidemiology of adult asthma in Asia: Toward a better understanding. Asia Pac Allergy 2014;4:75-85.

3. Loftus PA, Wise SK. Epidemiology of asthma. Curr Opin Otolaryngol Head Neck Surg 2016;24:245-9.

4. Sembajwe G, Cifuentes M, Tak SW, Kriebel D, Gore R, Punnett L, et al. National income, self-reported wheezing and asthma diagnosis from the world health survey. Eur Respir J 2010;35:279-86.

5. Wijesinghe M, Weatherall M, Perrin K, Crane J, Beasley R. International trends in asthma mortality rates in the 5-to 34-year age group: A call for closer surveillance. Chest 2009;135:1045-9.

6. Tabeshour J, Zirak MR, Mirakhorli H, Bazzaz BS, Afzalaghaee M, Kerachian $\mathrm{M}$, et al. Community pharmacist attitude toward collaboration with physicians. Int J Pharm Pharm Sci 2017:9:212-7.

7. Cheema E, Sutcliffe P, Singer DR. Community pharmacist-led new medicine service for patients with a long term medical condition: A cross-sectional study. Int J Pharm Pharm Sci 2017;9:129-33.

8. Garcia-Cardenas V, Armour C, Benrimoj SI, Martinez-Martinez F, Rotta I, Fernandez-Lilimos F. Pharmacists interventions on clinical asthma outcomes: A systematic review. Eur Respir J2016;47:1134-43.

9. Mehuys E, Van Bortel L, De Bolle L, Van Tongelen I, Annemans L, Remon JP, et al. Effectiveness of pharmacist intervention for asthma control improvement. Eur Respir J 2008;31:790-9.

10. Okumura LM, Rotta I, Correr CJ. Assessment of pharmacist-led patient counseling in randomized controlled trials: A systematic review. Int $\mathrm{J}$ Clin Pharm 2014;36:882-91.

11. Robert C Weisell. Body mass index as an indicator of obesity. Asia Pac J Clin Nutr 2002;11:S681-4.

12. Global Initiative for Asthma, Global Strategy for Asthma Management and Prevention. Strategy Report; 2016.

13. Tran HT, Pham XK, Bui TH. Reliability of Vietnamese version of mini AQLQ (mini asthma quality of life questionaire). Ho Chi Minh City Med J 2018;22:119.

14. Juniper EF, Guyatt GH, Cox FM, Ferrie PJ, King DR. Development and validation of the mini asthma quality of life questionnaire. Eur Respir J 1999;14:32-8

15. Bereznicki BJ, Peterson GM, Jackson SL, Walters H, Fitzmaurice K, Gee $\mathrm{P}$, et al. Pharmacist-initiated general practitioner referral of patients with suboptimal asthma management. Pharm World Sci 2008;30:869-75.

16. Armour C, Bosnic-Anticevich S, Brillant M, Burton D, Emmerton L, Krass I, et al. Pharmacy asthma care program (PACP) improves outcomes for patients in the community. Thorax 2007;62:496-502.

17. Plaza V, Peiró M, Torrejón M, Fletcher M, López-Viña A, Ignacio JM, et al. A repeated short educational intervention improves asthma control and quality of life. Eur Respir J 2015;46:1298-307.

18. Shanmugam S, Varughese J, Nair MA, Balasubramanian R, Velu S,
Bhojan C, et al. Pharmaceutical care for asthma patients: A Developing country's experience. J Res Pharm Pract 2012;1:66-71.

19. Wang KY, Chian CF, Lai HR, Tarn YH, Wu CP. Clinical pharmacist counseling improves outcomes for Taiwanese asthma patients. Pharm World Sci 2010;32:721-9.

20. Petkova VB. Pharmaceutical care for asthma patients: A community pharmacy-based pilot project. Allergy Asthma Proc 2008;29:55-61.

21. Brown R. Behavioral issues in asthma management. Allergy Asthma Proc 2001;22:67-9.

22. Onda M, Sakurai H, Hayase Y, Sakamaki H, Arakawa Y, Yasukawa F, et al. Effects of patient-pharmacist communication in the treatment of asthma. Yakugaku Zasshi 2009;129:427-33.

23. Klijn SL, Hiligsmann M, Evers SM, Roman-Rodriguez M, van der Molen T, Van Boven JF. Effectiveness and success factors of educational inhalertechnique interventions in asthma and COPD patients: Asystematic review. Eur Respir J 2016;48:OA1998.

24. Foot H, La Caze A, Baker P, Cottrell N. Better understanding the influence and complexity of beliefs on medication adherence in asthma. Patient Educ Couns 2019;102:564-70.

25. Axtell S, Haines S, Fairclough J. Effectiveness of various methods of teaching proper inhaler technique. J Pharm Pract 2017;30:195-201.

26. Holtz B, Whitten P. Managing asthma with mobile phones: A feasibility study. Telemed J E Health 2009;15:907-9.

27. Lv Y, Zhao H, Liang Z, Dong H, Liu L, Zhang D, et al. A mobile phone short message service improves perceived control of asthma: A randomized controlled trial. Telemed J E Health 2012;18:420-6.

28. Pinnock H, Slack R, Pagliari C, Price D, Sheikh A. Understanding the potential role of mobile phone-based monitoring on asthma selfmanagement: Qualitative study. Clin Exp Allergy 2007;37:794-802.

29. Nelson P, Young HN, Knobloch MJ, Griesbach SA. Telephonic monitoring and optimization of inhaler technique. Telemed J E Health 2011;17:734-40

30. Benavides S, Rodriguez JC, Maniscalco-Feichtl M. Pharmacist involvement in improving asthma outcomes in various healthcare settings: 1997 to present. Ann Pharmacother 2009;43:85-97.

31. Saini B, Krass I, Smith L, Bosnic-Anticevich S, Armour C. Role of community pharmacists in asthma Australian research highlighting pathways for future primary care models. Australas Med J 2011;4:190-200.

32. Trinh HT, Nguyen HT, Pham VT, Ba HL, Dong PT, Cao TT, et al. Hospital clinical pharmacy services in Vietnam. Int J Clin Pharm 2018:40:1144-53. 
SUPPLEMENTAL TABLES

Supplemental Table 1: Effect of an intervention program on total QoL scores after 3-month follow-up with ITT analysis ( $\mathrm{n}=342$ )

\begin{tabular}{|c|c|c|c|c|}
\hline \multirow[t]{2}{*}{ Factor } & \multirow[t]{2}{*}{ Beta } & \multicolumn{2}{|l|}{$95 \% \mathrm{CI}$} & \multirow[t]{2}{*}{ p-value } \\
\hline & & Upper & Lower & \\
\hline Intervention (yes) & 0.474 & 0.830 & 1.214 & $<0.001$ \\
\hline Age & 0.016 & -0.006 & 0.008 & 0.743 \\
\hline Gender (Male) & 0.015 & -0.167 & 0.235 & 0.741 \\
\hline Education level & 0.057 & -0.090 & 0.346 & 0.249 \\
\hline \multicolumn{5}{|l|}{ (University/College/Postgrad) } \\
\hline Allergic rhinitis/sinusitis (yes) & 0.106 & -0.053 & 0.556 & 0.105 \\
\hline GERD (yes) & 0.013 & -0.202 & 0.262 & 0.799 \\
\hline Allergy (yes) & 0.211 & 0.267 & 0.703 & $<0.001$ \\
\hline Comorbidities (yes) & -0.078 & -0.638 & 0.169 & 0.253 \\
\hline Asthma severity & 0.084 & -0.006 & 0.199 & 0.066 \\
\hline Over weight/Obesity (yes) & 0.081 & -0.020 & 0.377 & 0.077 \\
\hline $\begin{array}{l}\text { Baseline inappropriate inhaler } \\
\text { technique }\end{array}$ & 0.073 & -0.054 & 0.599 & 0.101 \\
\hline
\end{tabular}

GERD: Gastroesophageal reflux disease, CI: Confidence interval

Supplemental Table 2: Baseline characteristics of patients lost to follow-up and remaining after 3 months in the CG (n=161)

\begin{tabular}{|c|c|c|c|c|}
\hline \multirow[t]{2}{*}{ Characteristics } & \multirow[t]{2}{*}{ Overall $(n=161)(\%)$} & \multicolumn{2}{|l|}{ Group } & \multirow[t]{2}{*}{ p-value } \\
\hline & & $\begin{array}{l}\text { Loss to follow-up } \\
\text { CG }(n=65)(\%)\end{array}$ & $\begin{array}{l}3^{\text {rd }} \text { month remaining } \\
\text { CG }(n=96)(\%)\end{array}$ & \\
\hline Female & 69.6 & 73.8 & 66.7 & 0.331 \\
\hline Mean Age & $43.36 \pm 14.33$ & $43.48 \pm 15.74$ & $43.27 \pm 13.37$ & 0.935 \\
\hline $18-60$ & 83.9 & 78.5 & 87.5 & 0.126 \\
\hline$\geq 60$ & 16.1 & 21.5 & 12.5 & \\
\hline BMI $\left(\mathrm{kg} / \mathrm{m}^{2}\right)$ & $22.64 \pm 2.80$ & $22.17 \pm 2.34$ & $22.95 \pm 3.04$ & 0.155 \\
\hline Over-weight/Obesity & 40.4 & 30.8 & 46.9 & 0.05 \\
\hline \multicolumn{5}{|l|}{ Education level } \\
\hline $\begin{array}{l}\text { Primary/secondary/High } \\
\text { school }\end{array}$ & 68.3 & 69.2 & 67.7 & 0.839 \\
\hline University/College/Postgrad & 31.7 & 30.8 & 32.3 & \\
\hline Asthma severity (GINA 2016) & $3.18 \pm 1.01$ & $3.29 \pm 1.02$ & $3.10 \pm 0.99$ & 0.262 \\
\hline \multicolumn{5}{|l|}{ Comorbidities } \\
\hline Yes & 81.1 & 74.1 & 85.4 & 0.094 \\
\hline Allergy & 34.2 & 32.3 & 35.4 & 0.683 \\
\hline $\begin{array}{l}\text { Inappropriate inhaler } \\
\text { technique }\end{array}$ & 91.1 & 90.5 & 91.5 & 0.827 \\
\hline Baseline QoL scores & $4.43 \pm 0.89$ & $4.40 \pm 0.85$ & $4.45 \pm 0.92$ & 0.588 \\
\hline
\end{tabular}

Mean age, body mass index, and asthma were compared between the two groups using the t-test. Rates of gender, age groups, overweight/obesity patients, education level, comorbidities, and inappropriate inhaler technique were compared using the Chi-square test. BMI: Body mass index, GERD: Gastroesophageal reflux disease 